\title{
Computer aided music therapy evaluation: Testing the Music Therapy Logbook prototype 1 system ${ }^{\text {th }}$
}

\author{
Elaine Streeter, PhD ${ }^{\mathrm{a}, *}$, Matthew E.P. Davies, $\mathrm{PhD}^{\mathrm{b}, 1}$, Joshua D. Reiss, $\mathrm{PhD}^{\mathrm{b}}$, Andy Hunt, $\mathrm{PhD}^{\mathrm{a}}$, \\ Richard Caley, Clinical Physicist ${ }^{\mathrm{c}}$, Cath Roberts, PG Dip Music Therapy ${ }^{\mathrm{d}, 2}$ \\ a University of York, United Kingdom \\ b Queen Mary University of London, United Kingdom \\ c Mid-Yorkshire Hospitals NHS Trust, United Kingdom \\ d Sheffield Teaching Hospitals NHS Trust, United Kingdom
}

\section{A R T I C L E I N F O}

\section{Keywords:}

Music therapy

Evidence based practice

Evaluation

Music information retrieval

Computer

Music analysis

\begin{abstract}
A B S T R A C T
Research indicates that music therapists are likely to make use of computer software, designed to measur changes in the way a patient and therapist make use of music in music therapy sessions. A proof 0 concept study investigated whether music analysis algorithms (designed to retrieve information fron commercial music recordings) can be adapted to meet the needs of music therapists. Computationa music analysis techniques were applied to multi-track audio recordings of simulated sessions, then tc recordings of individual music therapy sessions; these were recorded by a music therapist as part of he ongoing practice with patients with acquired brain injury.

The music therapist wanted to evaluate two hypotheses: one, whether changes in her tempo wer affecting the tempo of a patient's play on acoustic percussion instruments, and two, whether her musica interventions were helping the patient reduce habituated, rhythmic patterning. Automatic diagram were generated that gave a quick overview of the instrumental activity contained within each session when, and for how long each instrument was played. From these, computational analysis was applied t musical areas of specific interest. The results of the interdisciplinary team work, audio recording tests computer analysis tests, and music therapy field tests are presented and discussed.
\end{abstract}

(C) 2011 Elsevier Inc. All rights reserved
The purpose of the proposed system is to help therapists keep track of what happens in music therapy, taking into account changes in music; so that changes in music can be objectively monitored (and quantified) in relation to other types of information, such as descriptive, written notes. The long term aim is to provide therapists with an everyday practice evaluation tool, which can be used to write session notes, record music therapy sessions, and analyse music data (Streeter, 2010, pp. 195-196).

\footnotetext{
This research was supported by a Music Therapy Charity Research Fellowship awarded to Elaine Streeter, and a White Rose Health Technology Innovation Proof of Concept award to Elaine Streeter and Andy Hunt of the University of York, Joshua D. Reiss of Queen Mary University of London, Richard Caley of the Mid-Yorkshire NHS Trust and Cath Roberts of the Sheffield Teaching Hospitals NHS Foundation Trust.

* Corresponding author at: Honorary Research Fellow in Music Therapy, Department of Music, University of York, Heslington, York YO10 5DD, United Kingdom. Tel.: +4407786155451.

E-mail address: es530@york.ac.uk (E. Streeter).

1 Present address: INESC Porto - Instituto de Engenharia de Sistemas e Computadores do Porto, Portugal.

2 Present address: Sheffield Health and Social Services NHS Trust, United Kingdom.
}

The study follows from previous collaborations between musi therapists and engineers. Since the 1990s, music therapists have made good use of computers and computational analysis to assis them in their research (Streeter, 2007). The first music therapis to trial computational analysis of music data from music therapy sessions was UK therapist, Mary Abbotson. Abbotson co-developec the Computer Aided Music Therapy Analysis prototype with engineers Dr Andy Hunt, Dr Adrian Verity, Mark Hildred, and musi therapist, Felicity North (Hunt, Kirk, Abbotson, \& Abbotson, 2000 Verity, 2003). In Finland, Professor Jaako Erkilla has been devel oping and testing the Music Therapy Toolbox system - a research tool that analyses MIDI data produced when patients and therapists improvise on MIDI instruments (Erkkilä, 2007). In Israel, D Avi Gilboa has been developing The Map (Gilboa, 2007), a compute based notation system for mapping music therapy sessions. In addi tion, Benveniste, Jouvelot, Lecourt, and Michel (2009) integratec Wii technology within a prototype digital musical instrument along with French music therapist, Edith Lecourt.

The Music Therapy Logbook approach differs from those above in that it is the first to apply computational music analysis to recordings of individual music therapy sessions in which acoustic percussion instruments were played simultaneously with a MID 
piano. The approach posed a set of technical challenges: unlike MIDI data, audio recorded from acoustic instruments is subject to interference from other sounds occurring in or around a music therapy room. However, to meet the needs of practicing music therapists it was decided to work towards delivering a system that can cope with such challenges (many music therapists prefer, or only have access to, acoustic instruments).

The focus of this paper is therefore to present the first set of test results generated by the Music Therapy Logbook Proof of Concept Study. The researchers used existing recording equipment and applied existing algorithms; these were adapted to generate information relevant to music therapy evaluation. The paper discusses the results of tests designed to find, identify changes in, and keep count of musical events across a series of individual music therapy sessions. The recording method was tested by a music therapist working with patients in a neuro-rehabilitation unit. The analysis tests were performed by a computer engineer in conjunction with a music therapist. Further research and development is necessary before The Music Therapy Logbook system can be made available to music therapists, however, initial software interface development (for Music Therapy Logbook analysis software) has been undertaken; a description of the preliminary software interface design is published elsewhere (Streeter, 2010, pp. 194-2011).

\section{Method}

\section{Inter-disciplinary collaboration}

All stages of the research required collaboration between disciplines. The team included three engineers, a clinical physicist and two music therapists. It was important to acknowledge that given the high levels of technical expertise available, computer engineering could wield more influence than music therapists' opinions. It was therefore important that music therapy was illustrated to the team via audio and video material, in order to establish the types of musical events music therapists want to evaluate.

Understandably, because engineers are not clinicians, the team struggled at first to agree the purpose of a practice evaluation tool; that it differs from that of a tool devised to assess improvement in a patient's condition. A key factor that guided the team was that music therapists set patient centred goals, but that these can range from psycho-social goals to restorative goals; for example supporting a patient through emotional adjustment to injury, as compared with helping a patient recover skills (Daveson, Magee, Crewe, Beaumont, \& Kenealy, 2007). As the unifying factor across all music therapy approaches is the developing relationship between therapist and patient, it was established that the research should prove the concept of tracking musical changes that take into account the patient's musical expression in relation to the therapist's musical expression. The analysis tests were designed to answer real evaluation questions provided by working music therapists. To enhance the research, a focus group was consulted. In addition, four surveys were carried out ( $n=6, n=10, n=44, n=125$ ). The results of the user opinion research are published in full elsewhere (Streeter, 2010, pp. 92-112).

\section{Audio recording technique}

A multi-channel, wireless, digital audio recording system was assembled from readily available, off the shelf products. Small contact microphones were tested; these were individually attached to each musical instrument. The microphones (with their individual transmitters) were attached in such a way as not to impede performance; for example, a microphone was attached to the inside of a snare drum head. By this means audio spill from one instrumental
Table 1

Simulated test recordings: set 1 : conga drum duet.

\begin{tabular}{|c|c|}
\hline Improvisation number & $\begin{array}{l}\text { Simulated music therapy improvisation: } \\
\text { description }\end{array}$ \\
\hline Set $1: 1$ & $\begin{array}{l}\text { Patient does not play instrument - therapist plays } \\
\text { to patient. }\end{array}$ \\
\hline Set $1: 2$ & $\begin{array}{l}\text { Patient makes fleeting sounds on drum then long } \\
\text { silences - therapist offers musical support. }\end{array}$ \\
\hline Set $1: 3$ & $\begin{array}{l}\text { Patient makes fleeting sounds, reduces silences - } \\
\text { therapist continues as above. }\end{array}$ \\
\hline Set $1: 4$ & $\begin{array}{l}\text { Patient occasionally plays unstable tempi - } \\
\text { therapist supports patient's tempi. }\end{array}$ \\
\hline Set $1: 5$ & $\begin{array}{l}\text { Patient rarely establishes tempi - but the players } \\
\text { engage in some simultaneous play. }\end{array}$ \\
\hline Set $1: 6$ & $\begin{array}{l}\text { Patient's tempo established more frequently - } \\
\text { therapist matches patient's tempo. }\end{array}$ \\
\hline Set $1: 7$ & $\begin{array}{l}\text { Patient tempo fully established and sustained - } \\
\text { therapist sustains and matches tempo. }\end{array}$ \\
\hline Set $1: 8$ & $\begin{array}{l}\text { Therapist changes tempo }-\backslash \text { patient does not } \\
\text { match therapist's change in tempo. }\end{array}$ \\
\hline Set $1: 9$ & $\begin{array}{l}\text { Patient initiates tempo changes - therapist } \\
\text { responds through imitation. }\end{array}$ \\
\hline Set $1: 10$ & $\begin{array}{l}\text { Patient's tempo imitates changes in the therapist's } \\
\text { tempo. }\end{array}$ \\
\hline Set $1: 11$ & $\begin{array}{l}\text { Patient plays rhythmic patterns - waits whilst } \\
\text { therapist responds with own rhythmic patterns. }\end{array}$ \\
\hline Set $1: 12$ & $\begin{array}{l}\text { Patient and therapist respond to each other's } \\
\text { tempo changes, are able to imitate and initiate } \\
\text { rhythmic patterns. }\end{array}$ \\
\hline
\end{tabular}

track to another track was limited (thus it was possible for each instrumental track to be separately analysed by the computer).

By using this method players can change instruments during a music therapy session, and move about in the normal way, without being restricted by leads or wires, or limited by the positioning of MIDI percussion instruments (which need to be conjoined with a computer). Audio signals were transmitted to a small, portable, multi-channel receiver device; this was located away from the activity area, plugged to a laptop computer. The laptop ran existing audio recording software (Ableton Live 7 was used in this instance). The sessions were therefore recorded straight to a laptop computer.

\section{Simulating music therapy events}

Although the music therapists were keen to stress that musical progress, of itself, cannot provide evidence of meaningful change in music therapy, it was clear that the computer engineers needed a progressive sequence of music events to test whether music information retrieval (MIR) techniques could identify and track changes in music over time. Therefore, graded examples of musical exchanges between music therapist and patient were simulated. The instructions for these improvisations were designed to illustrate possible changes in a patient's and therapist's music play over 12 weeks. One player improvised from the therapist's point of view, the other from the patient's perspective. Thus, these simulated test recordings provided idealised examples of changes in music within a music therapy relationship; for example, a patient with a fixed tempo showing awareness of a therapist's change of tempo by speeding up and matching it.

Six improvisation sets were performed and recorded. A different instrumental combination was used for each set. Set 1 required two conga drums with each player playing a separate drum. The second set involved one player performing on both conga drums, whilst the other performed on an acoustic grand piano. The remaining sets tested whether the recording technique was delivering sufficiently clean sound from specific instruments; for example a xylophone, a Sound Beam, and a guitar.

Table 1 describes the improvisation set during which the two players were limited to one conga drum per person. Each 
improvisation was performed to give evidence of a greater level of musical engagement on the part of a patient, and between patient and therapist. The simulated improvisations exemplified how at first a patient may not show awareness, then tentatively establish his or her tempo, then, through tempo imitation, show awareness of the therapist's tempo, and eventually exchange musical play with a therapist; whereby the two players and are able to imitate and initiate musical dialogues through interactive, rhythmic play.

\section{Collaborative research process}

Computational music analysis tests were applied to the simulated test recordings by specialist engineer, Dr Mathew Davies. The collaboration consisted of the lead music therapist defining the type of music analysis requested, the engineer then investigating the best method of achieving this; the music therapist was therefore guided by the expertise of the engineer who applied computer algorithms to the recorded test material. ${ }^{3}$ The researchers needed time to explain what was requested by one and achievable for the other.

Therefore, although music therapist's questions guided the aims of the computational analysis, the computational tests described here were achieved by a process of collaborative experimentation; technical limitations playing an important part in determining outcomes. The two researchers then met with the team to discuss the results of music therapy analysis tests, and to decide on the most appropriate next step.

\section{Clinical field test recordings}

Field test recordings were arranged at the neuro-rehabilitation unit of The Hawthorns residential long stay facility in Peterlee, County Durham, United Kingdom.

The majority of patients in the unit had enduring neurodegenerative illness, or were suffering brain damage from traumatic injuries. The clinical music therapist working in the unit was external to the research team, and an expert in the NordoffRobbins music therapy approach. ${ }^{4}$ This approach encourages the use of live improvisation.

Following a 2-h training session (in the unit) the music therapist was left with the recording equipment and a laptop computer. The therapist recorded individual music therapy sessions with four patients over a period of five weeks. The patients were mid way through their therapy and familiar with their sessions being audio recorded. During each session the therapist improvised using a MIDI piano, whilst the patient used a variety of tuned and un-tuned acoustic percussion instruments. The therapist was asked to fill out a report form for each session. The form asked for information on the aims of each session and to report on any difficulties in using the recording equipment.

After the test period, the clinical music therapist met with the research team to discuss practical issues arising from her use of the equipment, and to present the evaluation questions arising from her work with the patients; in particular questions arising from her work with one patient, who is referred to here as; $\mathrm{Mr} \mathrm{B}$.

\footnotetext{
3 These music information retrieval techniques were adapted from existing algorithms in use at the Centre for Digital Music, Queen Mary, University of London, at the time of the research.

4 The approach was not a requirement - rather it happened to be the method in use at this unit.
}

Mr B - history and presenting behaviour

Mr B was 52 years old and a long stay resident of the unit. He hac acquired a brain injury following a motorcycle accident in which he had suffered a fractured skull, fractures to his right arm, his right femur, right tibia and right fibula.

Mr B's brain injury had led to cognitive impairment and memory problems, although he was still able to use some expressive language. His mood was known to fluctuate and it had been reported that Mr B could become verbally aggressive. He ofter showed confusion and disorientation and was very reluctant to join in shared activities, spending most of the time by himself in his room. Mr B needed help to stand up; his hand dexterity anc fine motor control were poor, and he was a wheel chair user.

\section{Therapy aims}

During the test period the aims of the multi-disciplinary clinica team were twofold; to help improve the patient's mobility and to lessen his isolation by encouraging his interactive social skills. The music therapist was focusing on the latter aim; one objective was to help Mr B experience increased flexibility in his musical improvisations with her, in particular to help him reduce the number o times rhythmic phrases were repeated which, when played con tinuously, were thought by the therapist not to be intentionally communicative, but habituated.

The therapist reported that $\mathrm{Mr} \mathrm{B}$ thought his playing reflectec the way he behaved; "This is what I'm like" he said, "I always gc too fast". Through her musical interventions, the therapist aimec to widen the range of Mr B's tempi; in particular encouraging him to slow down. Mr B described how "Life is like music therapy, we make it up as we go along". So at the heart of the work with Mr. $\mathrm{E}$ was the patient's recognition that music therapy involves creative thinking; a shared process in which flexible, rather than fixed, ways of behaving can be tried out.

\section{Music therapy aims determine computational analysis tests}

It is important that computational techniques for analysing music therapy are investigated in relation to the needs and interests of music therapists. In this case, as the music therapist wa using the Nordoff-Robbins approach, her particular interest was in evaluating the effects of her improvised music on the way Mr B was able to make use of improvisation.

Therefore, the computational analysis tests were defined, in discussion with herself and the team, to reflect questions arising directly from her music work with $\mathrm{Mr} \mathrm{B}$. Two evaluation questions emerged from discussions with the therapist:

\section{A therapy process question:}

Can computational music analysis identify whether the therapist's tempo changes are effective in increasing the patient's tempo flexibility?

\section{A therapy progress question:}

Can computational music analysis quantify the amount o time the patient spends repeating a persistent rhythmic pattern?

\section{Computational music analysis of field test recordings}

The foundation of the computational analysis rested on the com puter being able to identify and automatically isolate regions o musical activity in each music therapy session. The process of find ing these regions is referred to here as music-silence segmentation The purpose of this segmentation was two-fold; first, it providec 


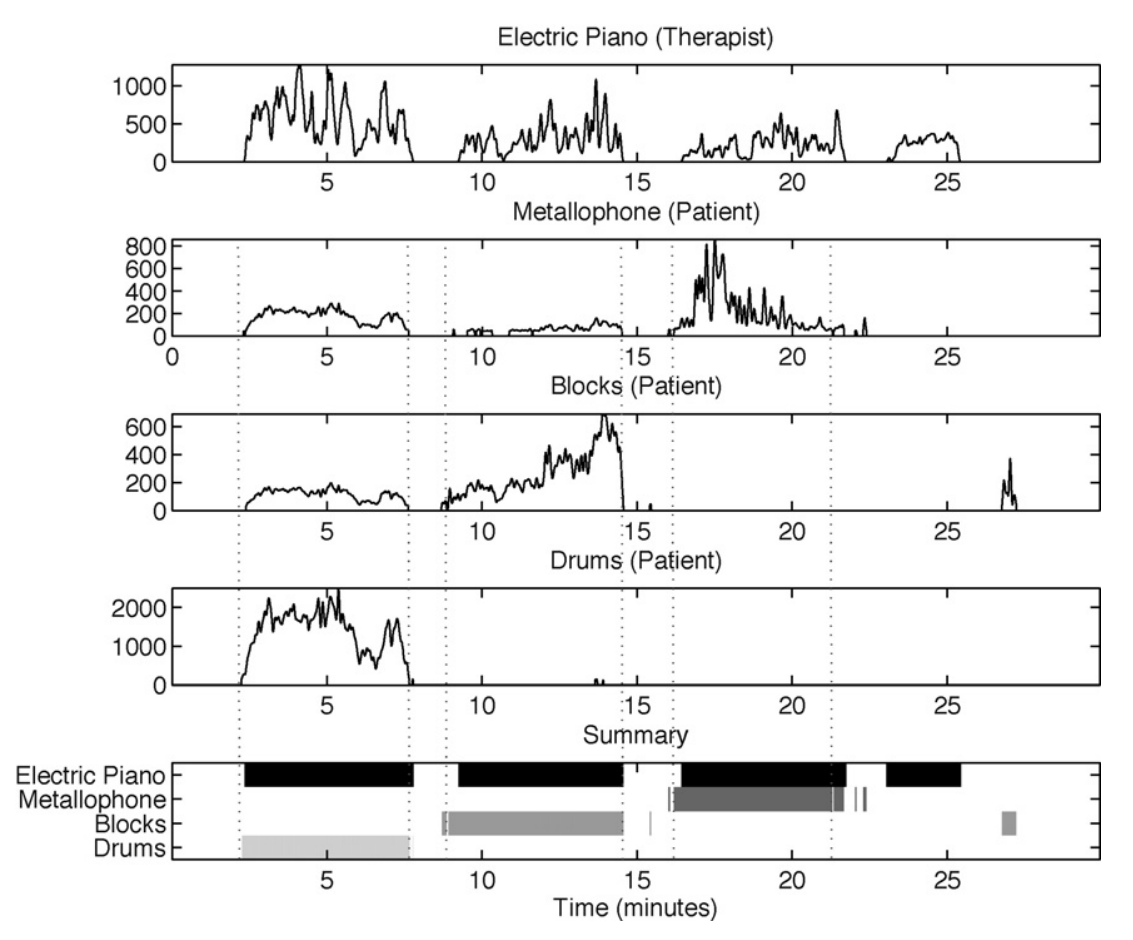

Fig. 1. Instrumental activity of therapist and $\mathrm{Mr} \mathrm{B}$ : test week 2 ( $Y$-axis: amplitude range/X-axis: time in min).

an important summary visualisation of a music therapy session, indicating which instruments had been played when, in what combination, and for how long. Second, by the computer knowing when each instrument had been played, any further computational analysis could be localised to specific regions of interest. An example of music-silence segmentation is shown in Fig. 1. It is clear from this that when the therapist and patient improvised music, they were creating it together. The instrumental summary at (e) shows that $\mathrm{Mr} B$ started playing the drum at $2.3 \mathrm{~min}$, that at 9 min into the session he was playing a set of wood blocks; only later did he play the metallophone.

The results shown in Fig. 1 also indicate that musical activity on one percussion instrument was picked up (at a lower level) by other microphones on other instruments; for example, the patient's drum playing is shadowed at a lower amplitude on the wood block track and the metallophone track (the MIDI piano outlet was connected directly to the computer so these signals were not affected). Therefore, in order to ensure that the correct instrumental track was selected for analysis, the audio channel with the greatest energy level was always chosen.

\section{Results}

\section{Simulated test recordings: improvisation set 1}

The focus here was on identifying and representing changes in instrumental activity over time. Fig. 2a shows how the ratios of playing to non-playing were represented by the computer. The top chart represents the therapist's activity, the middle chart represents that of the patient and the lowest chart represents the note onset consistency between players. ${ }^{5}$ From this it can be seen that the computer has identified, tracked and represented the increasing amount of instrumental activity from the player simulating

\footnotetext{
5 Onset consistency is a measure of the number of near-simultaneous events played by the therapist and patient. We defined a near-simultaneous event as one that occurs within $50 \mathrm{~ms}$ of another.
}

a patient. Similarly, the ratio of playing to non-playing from the therapist player is shown. Thus, a generalised snapshot of a (simulated) musical relationship, evolving over time, was generated. Fig. $2 \mathrm{~b}$ shows a bar chart representation of the same set of results. From this type of diagram it is possible to quickly identify sessions in which a patient and/or therapist increases their activity, and sessions in which their activity decreases.

Of particular interest is the dip in the note onset activity at test week eleven. In this improvisation the therapist player invited a drumming conversation: - the 'therapist' improvised a rhythmic phrase whilst the 'patient' listened, the 'patient' player responded by playing another phrase whilst the 'therapist' listened - thus there was less simultaneous activity. The graph illustrates how a marked decrease in note onset consistency in this case matched an increase in call and response type play.

In order to show how acoustic instrumental activity can be monitored over a series of sessions, instrumental activity diagrams were generated from the first 5 min of nine, simulated music therapy sessions. Fig. 3 shows how differing amounts of instrumental activity can be displayed quickly on the computer screen.

\section{Quantifying instrumental play: field test recordings: $\mathrm{Mr} B$ week 2}

By applying the same music-silence segmentation technique to the field test recordings, automatic identification of the regions of musical activity were generated. Thus it was possible to compute the percentage of session time during which $\mathrm{Mr} \mathrm{B}$ and the therapist played their instruments. Table 2 shows the percentage of session time spent playing instruments, and the duration of each person's play in test week 2 .

Automated tracking of tempo changes: field test recordings: $\mathrm{Mr} B$ week 2

Given the output of the music-silence segmentation analysis, the researchers were able to analyse regions of interest with the aim of extracting higher level musical information, matched to the therapist's evaluation questions. To enable comparisons between the 

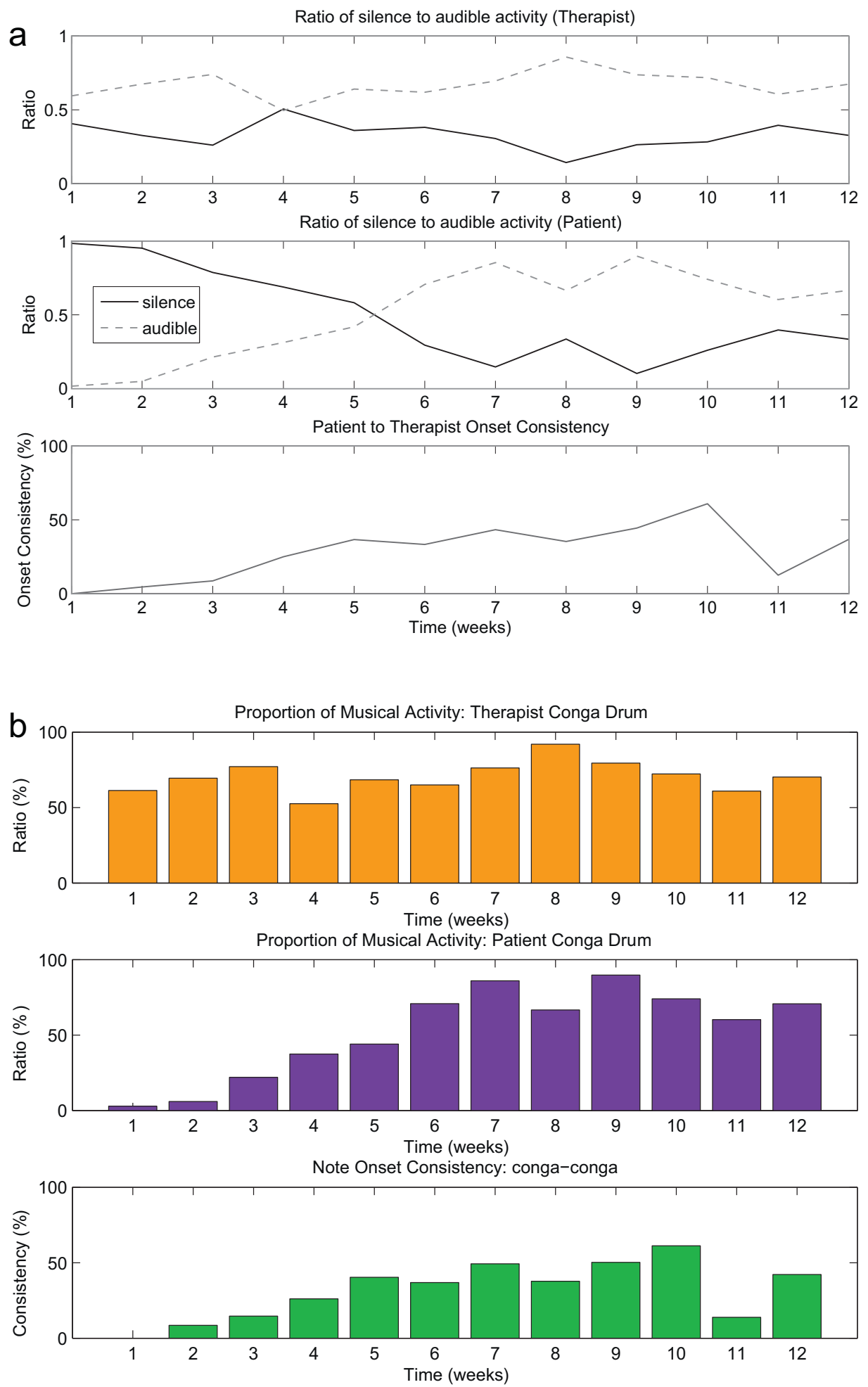

Fig. 2. (a) Analysis results: conga duet test: activity to silence ratios (solid lines represent silence, dashed lines represent conga playing activity). (b) Analysis results: cong duet test: activity bar chart.

tempo of the therapist and the patient, each channel was first analysed independently, then these results were combined into one summary visualisation. The process for identifying and tracking the tempo of each of the players' improvisation based on existing work in rhythm analysis (Bello, Duxbury, Davies, \& Sandler, 2004; Davies, 2007). The onset detection function was split up by the computer into analysis frames across the length of each region of musical activity. Each input frame was then automatically compared to a set of template functions covering a wide range of tempo hypothe ses (50-220 beats per minute). From this the computer extracted path of tempo through time (a tempo contour), examples of which for the therapist and the patient are shown in Fig. 4.

In each of the shared improvisations the analysis shows a clea downward trend in the extracted tempo contours; i.e. a distinc slowing down by therapist and patient within each of the instrumental improvisations during the session. 
Week 1

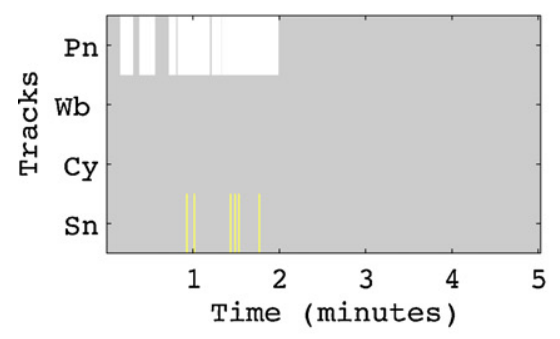

Week 4

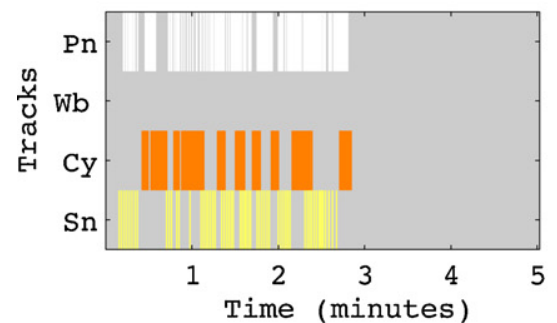

Week 7

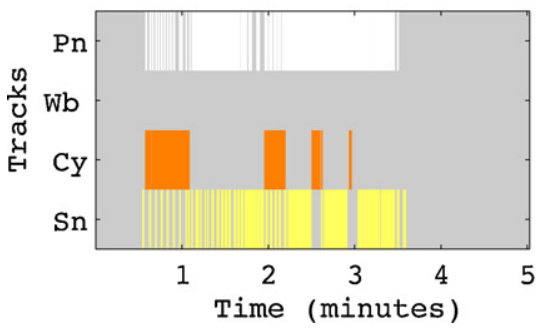

Week 2

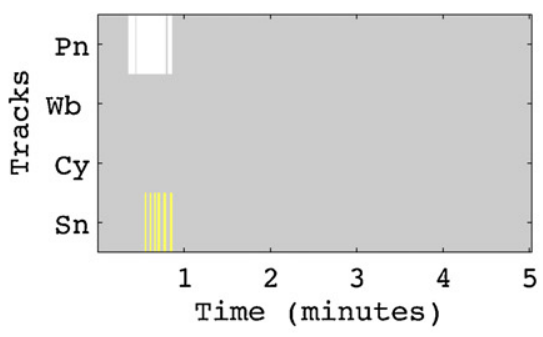

Week 5

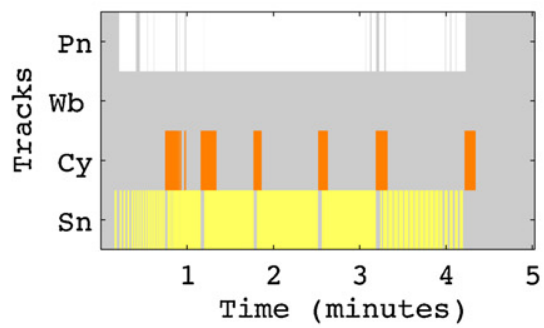

Week 8

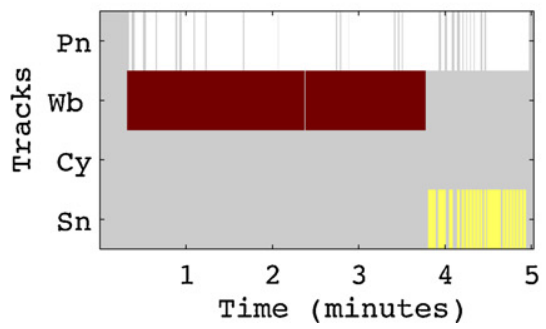

Week 3

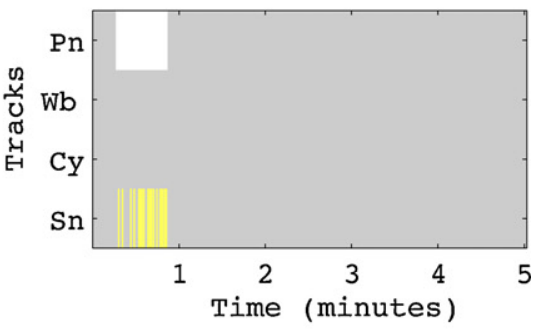

Week 6

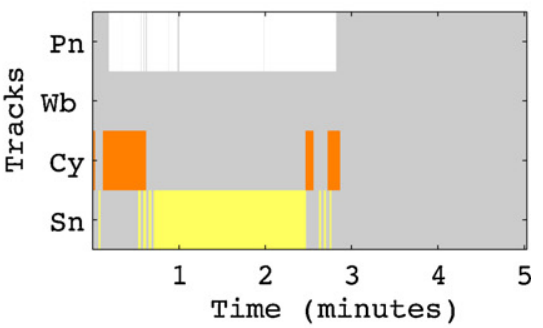

Week 9

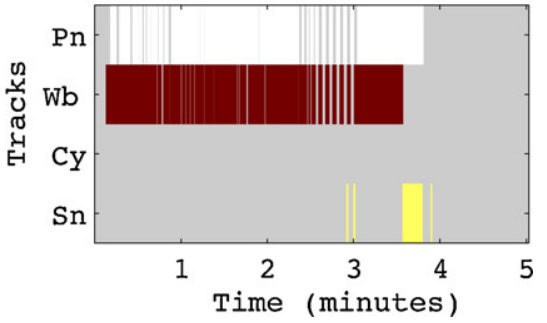

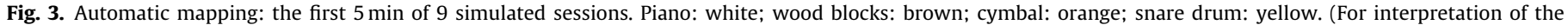
references to color in this figure legend, the reader is referred to the web version of the article.)

\section{Identification and quantification of rhythmic patterns: $M r$ B week} 2

The therapist had reported that $\mathrm{Mr} B$ repeatedly played a habituated phrase during their improvisations. The extraction of tempo therefore enabled higher level (more complex) computational analysis to be undertaken. The rhythm analysis was extended to identify beat locations (equivalent to human foot-taps in time to music). This information was then used to identify the repeated rhythmic pattern, described by the therapist as Mr B's 'jig' rhythm. A computer's recognition of beat locations allows analysis to operate in musical time, comparing the playing beat-by-beat rather than over fixed time scales. This is especially important as it enables meaningful analysis of rhythm even when tempo varies. Beat locations were extracted using a proven dynamic programming algorithm (Ellis, 2007).

An example plot is shown in Fig. 5.

Fig. 6 illustrates all extracted rhythmic patterns found to be present in Mr B's drum playing. Through inspection of these, and other patterns extracted from Mr B's wood block and metallophone play, those consistent with the 'jig' rhythm were identified, and the proportion of time Mr B spent playing the 'jig' rhythm over the whole session was computed.

Pattern 2 (Fig. 6) was identified as the 'jig' rhythm; having identified this, the computer was able to generate data measuring the occurrence of the pattern during each instrumental improvisation. The results are as follows: The 'jig' rhythm was played for $65 \%$ of the drum improvisation ( $3.25 \mathrm{~min}$ ), patterns 1 and 3 (Fig. 6) were not characterised by the 'jig' rhythm and took up the remaining $35 \%$ of the drum improvisation, none of the patterns played by $\mathrm{Mr} \mathrm{B}$ on the wood blocks were characterised by the 'jig' rhythm,
Table 2

Instrumental activity measurements: test week 2 .

\begin{tabular}{lll}
\hline Instrument & $\begin{array}{l}\text { Percentage of session } \\
\text { used for playing }\end{array}$ & $\begin{array}{l}\text { Duration of play } \\
\text { (session lasted 30 min) }\end{array}$ \\
\hline MIDI piano & $61.9 \%$ & $18.5 \mathrm{~min}$ \\
Metallophone & $19.0 \%$ & $5.7 \mathrm{~min}$ \\
Wood blocks & $21.2 \%$ & $6.3 \mathrm{~min}$ \\
Side drum & $18.2 \%$ & $5.4 \mathrm{~min}$ \\
Total therapist & $61.9 \%$ & $18.5 \mathrm{~min}$ \\
Total patient & $58.4 \%$ & $17.4 \mathrm{~min}$ \\
\hline
\end{tabular}

however, when Mr B played the metallophone, the analysis showed that he used two patterns which were both consistent with the 'jig' rhythm; these constituted 59\% (32+27\%) of the improvisation (3.36 min). Therefore, during the session in test week 2 , the patient was engaged in playing the 'jig' rhythm for $6.5 \mathrm{~min}$. (These results were then able to be compared with similar tests of rhythm analyses from other sessions, recorded during the research period with Mr B.)

\section{Discussion}

Audio recording technique

The proximity of the microphone to the sound source remains the most important factor in ensuring a high degree of audio separation on each instrumental track. Levels of audio separation vary according to which instrument is being recorded. By attaching microphones to each of the instruments, music therapists can adjust each audio input level to match the amplitude 

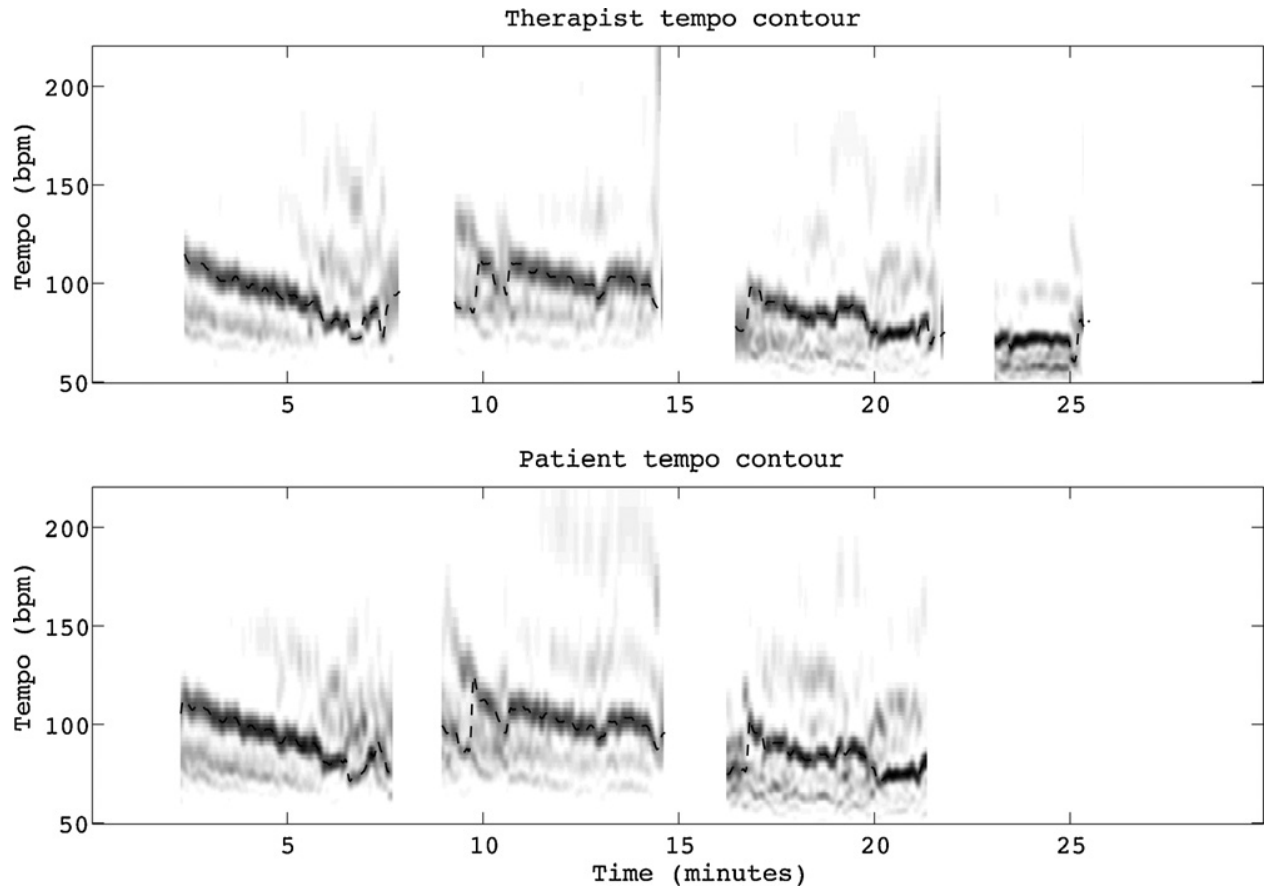

Fig. 4. Results of tempo contour analysis: test week 2. Therapist (upper plot), patient (lower plot) (bpm: beats per minute).

range of an instrument. A record of the input setting for each instrument can be kept (taking into account the acoustic of the music therapy room) so that these can quickly be adjusted at the start of the day. The input levels for each instrument can also be adjusted for different patients, so that if one person is known to play very quietly on a particular instrument, this can be taken into account.
The benefits of using a multi-track, mono recording technique are twofold; first it enables more accurate music computationa analysis as results are extracted from monophonic rather than polyphonic instrumental tracks, second; there are no microphone lead to impede movement. Within the limited time-frame and budget, $a$ reasonable level of audio separation was obtained using off the shel radio microphones. The inclusion of smaller microphones within

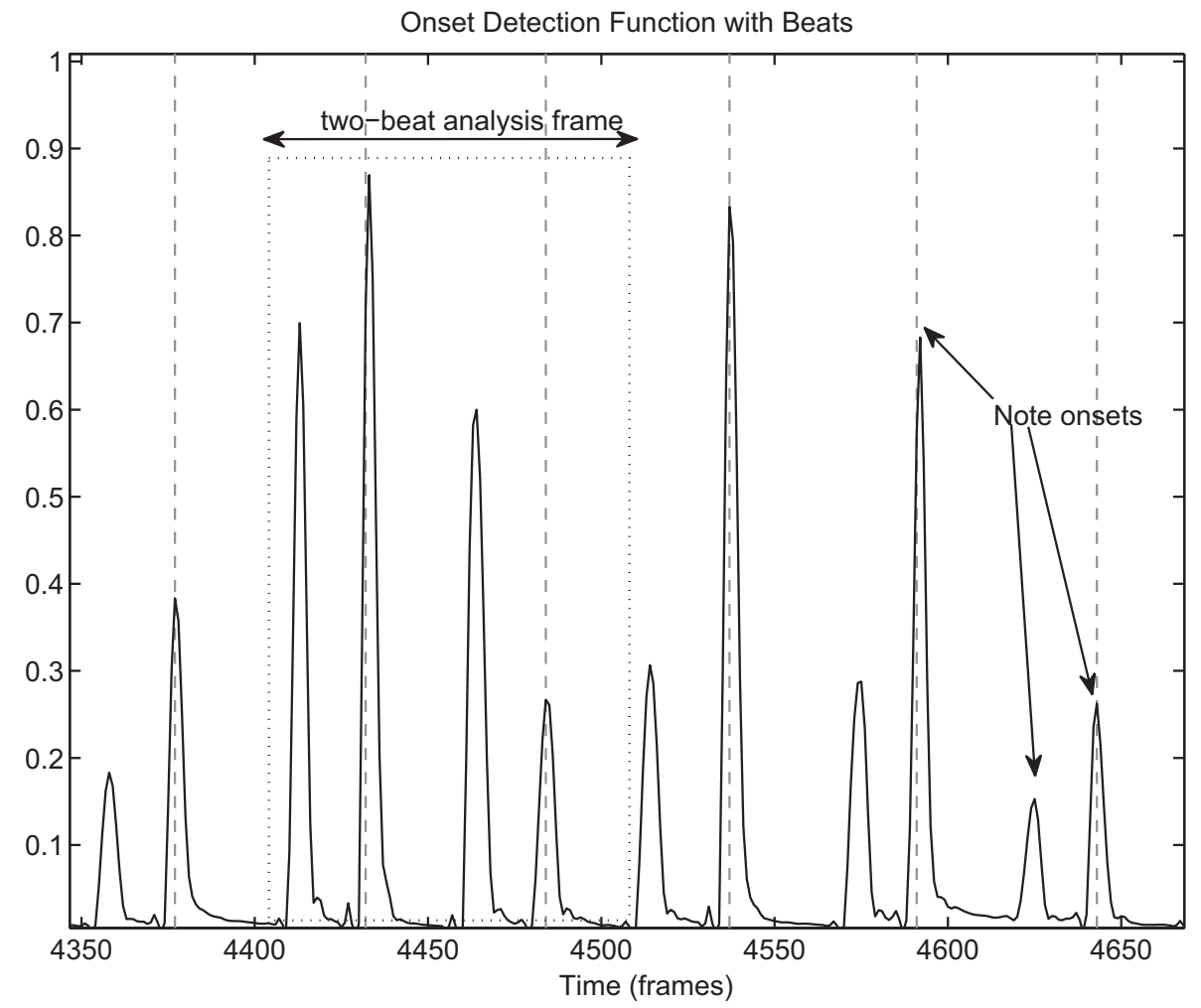

Fig. 5. Example of rhythmic pattern classification. Onset detection function with beat locations (shown as dotted vertical lines) +2 beat analysis frame. 
Pattern 1 (41\%)

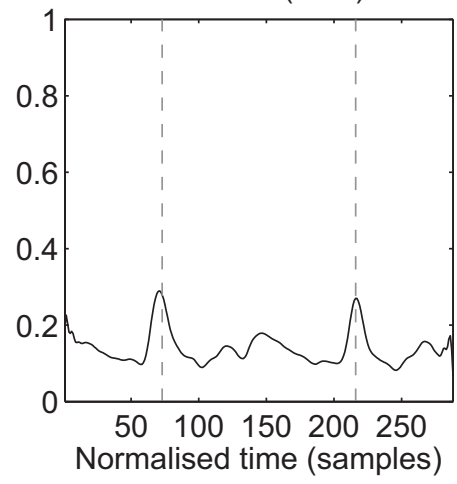

Pattern 2 (32\%)

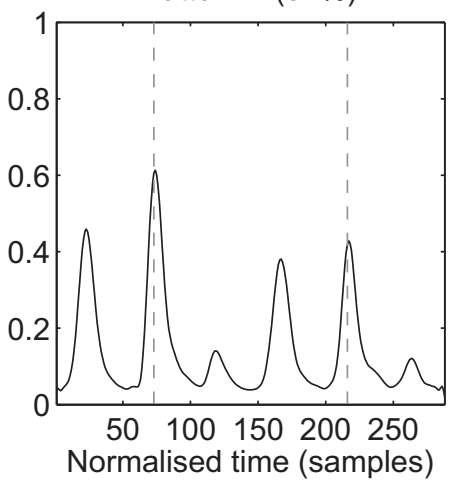

Pattern 3 (27\%)

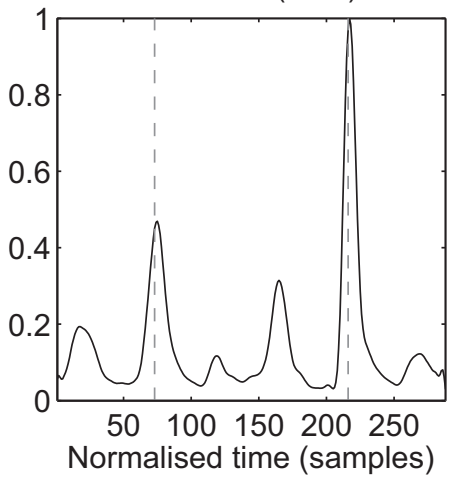

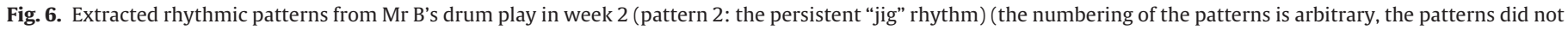
occur consecutively).

future system should facilitate even easier audio signal acquisition, and therefore lead to more complex areas of automatic analysis. A further area for investigation could be the use of automatic mixing techniques (Perez Gonzalez \& Reiss, 2011) and automatic audio source separation methods (Nesbit, 2007) to accentuate the separation of different instrumental channels.

\section{Simulated tests: identifying and measuring interaction events}

By using note onset detection techniques it was possible to identify call and response type play (see Fig. 2a and b). Therefore, in the future it is likely to be possible to refine this method to provide more detailed identification and measurement of interaction sequences. This finding is particularly important for future research, since music therapists have indicated a strong preference for analysis functions that can identify, measure, and quantify interaction sequences within music therapy improvisations: for example, $91 \%$ of British therapists surveyed ( $n=125)$ selected identification and quantification of interaction episodes as a particularly desirable function to be included in a future computer program, proposed to help them gather evidence (Streeter, 2010, p. 95).

\section{Field tests: mapping instrumental activity}

The results show computational analysis was able to deliver objective measurements of the amount of time a therapist and patient spent playing instruments (when acoustic instruments were used by the patient). This type of basic analysis provides useful information intended to help therapists keep track of what happens across a series of sessions. However, such measurements require interpretation by a therapist, taking into account the patient's history, the changing presentation of the patient's condition or illness, the aims of the therapy, and the context of care within the wider health team.

The importance of expert interpretation is understood by comparing Mr B's instrumental activity summary from week two with the therapist's prior knowledge of the patient: It is clear from the diagram (see Fig. 3 summary (e)) that the patient engaged in lengthy, simultaneous improvisations with the therapist. The therapist was already aware that $\mathrm{Mr} \mathrm{B}$ always played an instrument when she played, and that he found it very difficult to bring his improvisations to a close. Therefore, such results cannot provide evidence of positive communication or progress, they are merely neutral measurements of musical events that are only meaningful if used by the therapist (or, indeed, the patient) to review what has occurred. A therapist may, for example, want to question whether continuing to play when a patient cannot stop playing, is useful.
Another question arising is: who initiated the play? The results shown in Fig. 1 indicate that Mr B was the first to play during the woodblock/MIDI piano improvisation, he was also the first to play during the metallophone/MIDI piano improvisation. However, it cannot be argued from this that the patient initiated these improvisations with the therapist. Video analysis would be necessary to clarify whether, for example, the therapist may have looked at $\mathrm{Mr}$ $B$; such a glance could have been understood as a signal that he should start to play.

Therefore, the need for interpretation within the context of professional therapy is clearly established: measuring an increase in instrumental activity does not, in itself, evidence improvement; indeed, in the case of $\mathrm{Mr} \mathrm{B}$, a positive change would likely be evidenced by a decreasing amount of simultaneous engagement with the therapist.

\section{Field tests: tempo tracking}

It has long been established that timing and rhythm play an important role in developing social relationships (Stern, 1977; Trevarthen, 1999) and can be interpreted as an organising factor in music therapy processes (Streeter, 1979, 1981). Therefore, gaining access to objective evidence of changes in tempo is thought to be useful; therapists can monitor the results of their musical interventions; in the case of $\mathrm{Mr} \mathrm{B}$ reviewing whether a change from $4 / 4$ to $3 / 4$ time was effective in helping him experience tempo flexibility.

The results shown here evidence de-acceleration (see Fig. 4) and this was consistent with the therapist's aims, in as much as she wanted to help Mr B find ways of slowing his play on the acoustic percussion instruments. In this instance, the tempo pairing was so close that it was not possible to identify whose tempo was affecting who. However, it is noticeable that the tempo plots reflect something of the character of the musical relationship: when one player plays, the other plays, when one player slows, the other slows. Such data is likely to be helpful to music therapists when they want to assess changes in the music relationship, or explain the relevance of such changes to others.

\section{Field tests: rhythmic pattern identification}

The results show that Mr B was not limited to the 'jig' rhythm, although this was predominant (see Fig. 6). In fact, he had a number of rhythmic patterns in his repertoire, thus the results from test week 2 amplified the therapist's subjective observations. The computer was able to identify that the 'jig' rhythm did not occur when Mr B played the wood blocks, but that this style of playing was a strong feature of his use of other instruments. Being able to monitor 
the occurrence of patterning in relation to choice of instrument, and have a computer automatically locate improvisations (or sessions) in which such playing styles occur, was thought to be a very useful function for the future Music Therapy Logbook software. This opinion was supported by survey findings: $82 \%$ of UK music therapists $(n=125)$ selected this function as desirable (Streeter, 2010, p. 95). Why? The ability to initiate, repeat and imitate musical patterns are recognised stages of musical development (Ockenden, 2008). Such skills are known to be closely aligned to patterning in other modalities, such as social engagement, physical coordination, and the processing of language (Streeter, 2001).

\section{Performer identification}

After each therapy session the therapist reported which instruments had been played by the patient so that the researchers would know how to distribute the signals during the computational music analysis. The possible need for less time-consuming and more objective automatic performer identification is clearly raised. Taking into account practical limitations and ethical considerations, the team discussed a number of possible solutions to this; for example, integrating accelerometer technology into a wrist band, worn by the therapist. Existing medical aids, such as the Lifecomm emergency response device (http://www.lifecomm.com/devices.html) have already proven the use of such technology in monitoring spatial movements in relation to objects within a room. By making use of such technology, the computer will be helped to identify whether a particular sound corresponds to a movement by the therapist, or (by an absence of therapist movement) to that of a patient. Further opinions need to be gathered from music therapists working with different populations to understand how such technology may or may not meet ethical requirements. The need for further research is indicated in order to integrate such technology into the Music Therapy Logbook system.

\section{Summary of discussion}

In summary, the results show that when a patient improvises using acoustic percussion instruments in individual therapy sessions, it is possible to: (i) quantify the amount of time a patient spends in shared music making with a therapist, (ii) identify when a player starts or ends an improvisation, (iii) identify passages that may indicate musical dialogue, and (iv) display a general diagram of a patient's instrumental play with a therapist over a series of sessions. This data can then be used to support subjective reporting or help a therapist identify passages in which higher levels of computational analysis (e.g.; the analysis of tempo relationship) can be applied. In the case of Mr B's session analysis, identifying changes in tempo, and identifying rhythmic patterning, was successfully achieved; these tasks drew on proven music information retrieval techniques (Davies, 2007; Davies \& Plumbley, 2007).

Although the computer was able to identify one improvisation (out of a series of twelve simulated improvisations) in which call and response exchanges predominated, higher level questions about musical interaction have yet to be fully explored by music information retrieval (MIR) experts and will require a novel research effort. This research strand, however, presents an opportunity for computational music therapy analysis research to enhance investigations into the emergence of musical form, when players improvise rather than play from scores.

Whilst the success of the computational analysis was partially dependent on obtaining clean audio signals, an important aspect of the research was to determine if existing music information retrieval (MIR) techniques could be successfully applied to clinical music therapy recordings. The results presented here are encouraging, especially since this research is the first of its kind to analyse data derived from multi-track audio recordings of music therapy sessions in which acoustic percussion instruments were played.

\section{Conclusion}

The results show that by using computational music analysis, i is possible to: identify and map the duration of a patient's improvi sations on three different acoustic percussion instruments (whils a therapist plays a MIDI piano), compare this data to the duration and occurrence of the therapist's play, detect which player starts o ends an improvisation, and quantify the duration of a habituatec pattern played by a patient on two different acoustic percussion instruments. In addition, computational music analysis was suc cessfully used to track the tempo relationship between a patien and therapist. An important finding was that note onset detection was able to identify call and response type play that was known to predominate in one out of a series of 12 improvisations (simulated by the music therapy researchers to provide computer tes material).

The results of four surveys of music therapists indicate that for those therapists who already use computers at work, access tc such measurements is thought likely to be highly desirable; $91 \%$ o UK therapists who returned a survey questionnaire $(n=125)$ wan software that can identify and quantify interactive episodes from music therapy improvisations (Streeter, 2010, p. 95). By the com puter knowing in which part of an improvisation (in which session an increase or decrease in a particular type of instrumental play occurs, a therapist can quickly be directed to the point in the audic recording they want to listen back to. The computer can also store measurements of such events as they occur over a series of sessions and this data can be used to keep a record of changes in the musical relationship over time. One therapist commented, "This is the kind of stuff it is impossible to do in real life" another wrote; “ am already trying to do these things and it is very time consuming Great if a computer can help with the hard data" (Streeter, 2010, p 93).

The study also identified that, if a fully automatic system is found to be desirable, the need for performer identification tech nology (whereby a therapist's performance can be automatically distinguished from that of a patient) is indicated. The technology necessary to resolve this is already well established; for example micro-accelerometer technology, contained within personal med ical emergency devices worn on the wrist.

Therapists are expert in subjective reflection whilst comput ers are very good at identifying, gathering and quantifying data Changes in music may be significant in one music therapy relation ship, but bear no significance in another. It therefore remains the role of the therapist to interpret the data h/she asks a compute to extract from session recordings. Such data can then be used to support other types of evaluation; such as written notes, service users' feedback, and results of validated assessment tests, such a the Residual Music Skills Test (York, 2000). By keeping objective records of changes in musical relationships over time, therapist can both enhance their practice evaluation, and build up evidence of changes in music linked to diagnostic criteria, typical of different patient populations. The tests described here were not devisec in order to measure improvements in a patient's condition, but to capture relevant information matched to a therapist's evaluation questions about her work with a patient with acquired brain injury Thus, the Music Therapy Logbook approach aims to meet the need of practicing music therapists as well as researchers. A bespoke recording and music analysis system, that includes music therapy 
evaluation software, can help ensure that the evidence-base for music therapy remains musically informed.

\section{Acknowledgements}

The authors would like to thank: The Hawthorns Care and Neuro-Rehabilitation Unit, Peterlee, County Durham, Ms Janet Graham for testing the Logbook recording method in her live music therapy sessions, therapists at the Northern Ireland Music Therapy Trust for their focus group feedback, Dr Kathy Armour for research management advice, Mr Ray Beckett for his expert advice on audio recording techniques, and Professor Jane Edwards for her expert advice on music therapy evaluation.

\section{References}

Bello, J. P., Duxbury, C., Davies, M. E., \& Sandler, M. B. (2004). On the use of phase and energy for musical onset detection in the complex domain. IEEE Signal Processing Letters, 11(6), 553-556.

Benveniste, S., Jouvelot, P., Lecourt, E., \& Michel, R. (2009). Designing wiimprovisation for mediation in group music therapy with children suffering from behavioral disorders. In Proceedings of the 8th international conference on interaction design and children; design for health ACM SIGCHI, IDC'09 (pp. 18-26).

Daveson, B. A., Magee, W. L., Crewe, L., Beaumont, G., \& Kenealy, P. (2007). Music therapy assessment tool for low awareness states. International Journal of Therapy and Rehabilitation, 14(12), 545-549.

Davies, M. E. P. (2007). Towards automatic rhythmic accompaniment. PhD Thesis, Department of Electronic Engineering, Queen Mary, University of London.

Davies, M., \& Plumbley, M. (2007). Context-dependent beat tracking of musical audio. In IEEE Trans. Aud/Speech/Lang Proc., Vol. 15 (pp. 1009-1020).

Ellis, D. P. W. (2007). Beat tracking by dynamic programming. Journal of New Music Research, 36(1), 51-60.

Erkkilä, J. (2007). Music Therapy Toolbox (MTTB) an improvisation analysis too for clinicians and researchers. In T. Wosch, \& T. Wigram (Eds.), Microanalysis in music therapy: Methods, techniques and applications for clinicians, researchers, educators and students (pp. 134-148). London and Philadelphia: Jessica Kingsley Publishers.

Hunt, A., Kirk, R. Abbotson, M. \& Abbotson, R. (2000). Music therapy and electronic technology. Proceedings from EuroMicro conference, 2, 362-367.

Lifecomm Medical Emergency Device. Retrieved from http://www.lifecomm.com/ devices.html

Nesbit, A. L. (2007). Audio source separation by time-frequency masking. PhD Thesis, Department of Electronic Engineering, Queen Mary, University of London.

Ockenden, A. (2008). Music for children and young people with complex needs Oxford. p. 108.

Perez Gonzalez, E., \& Reiss, J. D. (2011). Automatic mixing. In U. Zölzer (Ed.), DAFX: Digital audio effects. John Wiley \& Sons, Ltd.

Stern, D. (1977). The first relationship. Harvard University Press.

Streeter, E. (1979). A theoretical background to the interpretation of rhythmic skills with particular reference to the use of music therapy as an aid to the clinical assessment of pre-school children. MMus Thesis, Dept of Music, University of York.

Streeter, E. (1981). Towards a theoretical understanding of rhythmic responses in music therapy. In British society of music therapy. Monograph. British Association of Music Therapy UK.

Streeter, E. (2001). Making music with the young child with special needs. London: Jessica Kingsley.

Streeter, E. (2007). Reactions and responses from the music therapy community to the growth of computers and technology some preliminary thoughts. In Voices: A world forum for music therapy. www.voices.no

Streeter, E. (2010). Computer aided music therapy evaluation: Investigating and testing the music therapy logbook prototype 1 system. PhD Thesis, Department of Music, University of York. http://etheses.whiterose.ac.uk/1201/.

Trevarthen, C. (1999-2000). Musicality and the intrinsic motive pulse: Evidence from human psychobiology and infant communication. In Rhythms, musical narrative, and the origins of human communication Musicae Scientiae, special issue. Liege: European Society for the Cognitive Sciences of Music., pp. $157-213$

Verity, A. (2003). A computer aided music therapy analysis system - CAMTAS. D.Eng Thesis, Department of Engineering, University of York.

York, E. F. (2000). A test-retest reliability study of the residual music skills test. Psychology of Music, 28(2), 174-180. 